\title{
Inflammation-induced lymphangiogenesis in the cornea arises from CD11b-positive macrophages
}

\author{
Kazuichi Maruyama, ${ }^{1}$ Masaaki li, ${ }^{2}$ Claus Cursiefen, ${ }^{1}$ David G. Jackson, ${ }^{3}$ Hiroshi Keino, ${ }^{1}$ \\ Minoru Tomita, ${ }^{1}$ Nico Van Rooijen, ${ }^{4}$ Hideya Takenaka, ${ }^{2}$ Patricia A. D'Amore, ${ }^{1}$ \\ Joan Stein-Streilein,, ${ }^{1,5}$ Douglas W. Losordo, ${ }^{2}$ and J. Wayne Streilein ${ }^{1}$
}

\begin{abstract}
1Ocular Immunology Group, The Schepens Eye Research Institute, Department of Ophthalmology, Harvard Medical School, Boston, Massachusetts, USA. 'Division of Cardiovascular Research, Caritas St. Elizabeth's Medical Center, Tufts University School of Medicine, Boston, Massachusetts, USA. ${ }^{3}$ Molecular Immunology Group, Institute of Molecular Medicine, University of Oxford, Oxford, United Kingdom. ${ }^{4}$ Department of Molecular Cell Biology and Immunology, Faculty of Medicine, Free University, Amsterdam, The Netherlands. ${ }^{5}$ Pulmonary and Critical Care, Brigham and Women's Hospital, Department of Medicine, Harvard Medical School, Boston, Massachusetts, USA.
\end{abstract}

\begin{abstract}
In the inflamed cornea, there is a parallel outgrowth of blood and lymphatic vessels into the normally avascular cornea. We tested whether adaptive and/or innate immune cells were actively involved in the genesis of new lymphatic vessels. Our results indicate that innate immune cells $\left(\mathrm{CD}_{11 b^{+}}\right.$macrophages, but not $\mathrm{CD} 11 \mathrm{c}^{+}$ dendritic cells) physically contributed to lymphangiogenesis under pathological conditions and that bone marrow-derived CD11b $b^{+}$acrophages expressed lymphatic endothelial markers such as LYVE-1 and Prox-1 under inflamed conditions in the corneal stromata of mice. Furthermore, blood vascular endothelial cells that expressed the Tie2 promoter did not contribute to newly formed lymphatic vessels under inflamed conditions. Our in vitro experiments demonstrated that $\mathrm{CD}_{11} \mathrm{~b}^{+}$macrophages alone were capable of forming tube-like structures that expressed markers of lymphatic endothelium such as LYVE-1 and podoplanin. The novel finding that $\mathrm{CD}_{11 \mathrm{~b}^{+}}$macrophages are critical for the development of inflammation-dependent lymphangiogenesis in the eye suggests a new mechanism of lymphangiogenesis.
\end{abstract}

\section{Introduction}

Lymphangiogenesis is an important milestone in tumor progression, and the extravasation of tumor cells via lymphatic vessels is thought to play a role in tumor dissemination. The lymphatic system also plays an essential role in the immune response to infectious agents. Afferent lymphatic vessels are the route by which APCs migrate to the regional lymph nodes and lymphoid organs.

The cornea is normally devoid of blood vessels and lymphatics; however, during inflammatory conditions such as penetrating keratoplasty (PKP), lymphatic vessels appear in the corneal stroma. We have previously suggested that lymphangiogenesis correlates with the corneal rejection reaction that occurs after PKP (1-3). Although the mechanisms that induce hemangiogenesis and lymphangiogenesis in the cornea after inflammation are not yet completely understood, newly recruited bone marrow-derived cells, such as macrophages, are known to produce angiogenic factors and therefore may play an important role (4).

Lymphatic vessels are often identified by electron microscopy. Ultrastructure characteristics of lymphatic vessels include the absence of luminal erythrocytes and a lack of abluminal pericytes. The lumens of lymphatic vessels appear collapsed and therefore are wide and thin and partly overlap the endothelial lining (5-7). While these features distinguish the structure of

Nonstandard abbreviations used: BVEPC, blood vascular endothelial progenitor cell; EGFP, enhanced green fluorescent protein; EPC, endothelial progenitor cell; PEC, peritoneal exudates cell; PKP, penetrating keratoplasty; TEM, transmission electron microscope.

Conflict of interest: The authors have declared that no conflict of interest exists.

Citation for this article: J. Clin. Invest. 115:2363-2372 (2005).

doi:10.1172/JCI23874. lymphatic vessels from blood vessels, a definitive method by which to identify lymphatic vessels based on their ultrastructure has yet to be developed (8). The lack of markers specific for the lymphatic endothelium has hampered the study of lymphangiogenesis. Recently, lymphatic markers such as VEGFR-3, podoplanin, prospero-related homeobox 1 (Prox-1), and lymphatic endothelium-specific hyaluronic acid receptor 1 (LYVE-1) were identified. Although VEGFR-3 is expressed by immature blood vessels and not by mature ones, it is constitutively expressed by the lymphatic endothelium (9-17). Bone marrow-derived

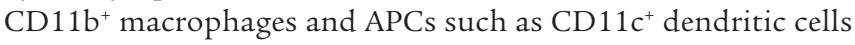
have been documented to be present in the corneal stroma (18). It is known that CD $11 \mathrm{~b}^{+}$macrophages produce VEGF-C and -D (4) and that the action of these cytokines on conjunctival lymphatic vessels via VEGFR-3 leads to the induction of lymphangiogenesis in the injured cornea (19). Moreover, some $\mathrm{CD}_{11} \mathrm{~b}^{+}$macrophages express LYVE-1 in the conjunctiva (20). Both VEGF-C and its receptor VEGFR-3 have been reported to be produced and expressed by activated macrophages $(21,22)$. Ligation of VEGFR-3 monocytes results in an immediate release of chemokines, an increase in the number of macrophages, and induction of hemangiogenesis and lymphangiogenesis. Based on these previous results, we postulated that activated macrophages have a critical role in the inflammation and pathogenesis that occurs after corneal transplantation.

We therefore examined the relationship between inflammation-induced pathological lymphangiogenesis and the immune cells' response in mice subjected to corneal transplantation or suturing. Since the healthy cornea is normally avascular but allows the growth of blood and lymphatic vessels during inflam- 

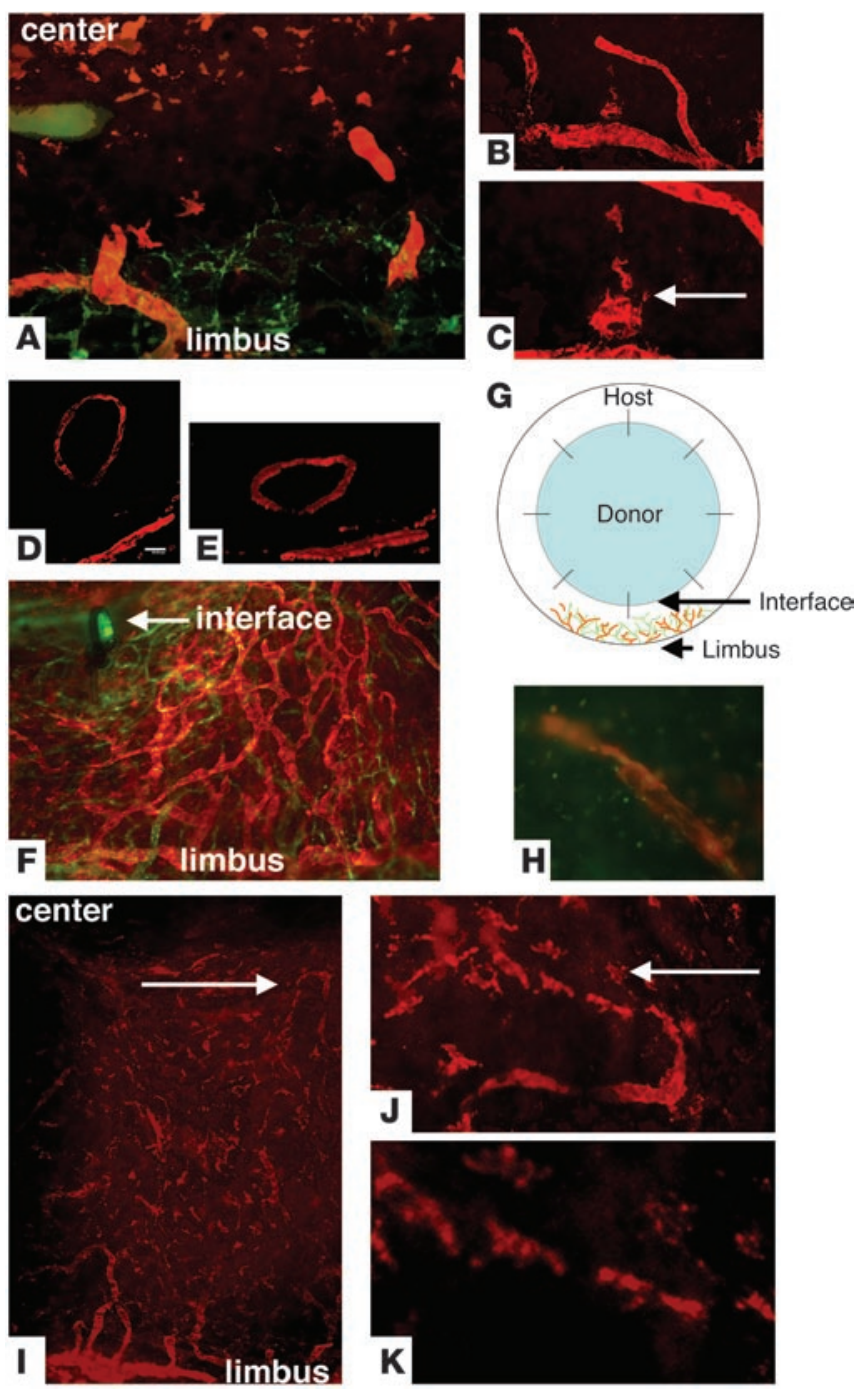

mation $(23,24)$, it is well suited for the study of hemangiogenesis and lymphangiogenesis. Lymphangiogenesis and hemangiogenesis have a critical role for antigen presentation and recruitment of the activated $\mathrm{T}$ cell to the site of attaching alloantigen. We found that inflammation-induced lymphangiogenesis sprouted from clusters of $\mathrm{CD} 11 \mathrm{~b}^{+}$cells in the center of the cornea rather than from the vessels in the limbus (periphery). These findings may be useful not only for studying rejection reactions after organ transplantation, but may also lead to a better understanding of tumor dissemination.

\section{Results}

Initial and late phases of lymphatic vessels in the corneal stroma. On day 3 after inflammation was induced in the cornea by PKP, we observed lymphatic vessels arising from the limbal area (Figure 1A). Unlike hemangiogenesis, in which lymphatic vessels can sprout from limbal vessels, the new lymphatic vessels appeared de novo to form from cell aggregates (Figure $1, \mathrm{~A}-\mathrm{C}$ ). LYVE- $1^{+}$cells in the vicinity of lymphatic vessels (Figure $1 \mathrm{C}$ ) appeared to coalesce with lymphatic vessels, raising the question as to how the lymphatic vessels are formed. Some of the lymphatic vessels did not have connections with limbal lymphatic vessels (Figure 1, D and E).

\section{Figure 1}

Initial (3-7 days) and late (56 days) phases of lymphangiogenesis after PKP. (A) Hemangiogenesis (green) and lymphangiogenesis (orange) in the corneal stromal layer 3 days after PKP. The blood vessel structures appear to originate from cellular aggregates. (B and $\mathbf{C}$ ) The dendritic-shaped cells aggregate and appear to anastomose with limbal lymphatic vessels 3 days after PKP. (D and E) A discrete lymphatic vessel (D) and its 3D image (E). (F) Lymphatic vessels reach the interface (arrow) between donor and host 7 days after PKP. (G) Diagram of eye structure after corneal transplantation showing location of blood vessels (green) and lymphatic vessels (orange). (H) LYVE-1 (orange) and FITC-conjugated CD31 (PECAM-1; green) staining of explanted culture cornea after stimulation with IL-1 $\beta$. (I-K) Fifty-six days after $\mathrm{PKP}$, the inflammation is suppressed, and lymphatic vessels regress. Arrows indicate interrupted structure and appearance of dendriticshape cells. Magnification, $\times 100$ (A, F, and I); $\times 200$ (B and J); $\times 400$ (C, H, and K). Scale bar: $40 \mu \mathrm{m}$ (D).

Seven days after corneal transplantation, we observed these lymphatic vessels in the central area of the cornea (Figure 1F). Figure $1 \mathrm{G}$ illustrates the increased corneal hemangiogenesis and lymphangiogenesis observed after corneal transplantation. We determined that the lymphatic vessels that formed in corneal explant cultures after inflammatory stimulation were formed by endogenous corneal and/or recruited cells. A corneal explant that consisted of the central $2-\mathrm{mm}$ portion of cornea (not including the limbal area) was stimulated with IL-1 $\beta$. Small structures (resembling lymphatic vessels) positive for CD31 and LYVE-1 were detected in central corneal explants cultured for 2-3 days. The development of these lymphatic-like structures was not continuous with that of limbal lymphatic vessels; rather, the new structures appeared to form de novo in the corneal tissue with limbal lymphatic vessels but appeared to form de novo in the corneal tissue (Figure $1 \mathrm{H}$ ).

During the late phase of inflammation (approximately 56 days after PKP in syngeneic transplantation), lymphangiogenesis appeared to cease, and the lymphatic vessels disassembled. The remaining LYVE-1 ${ }^{+}$cells (Figure 1I) appeared dendritic in nature (Figure $1, \mathrm{~J}$ and $\mathrm{K}$ ). In contrast to lymphatic vessels, some of the blood vessels induced under inflamed conditions were covered by dencytes and matured after the inflammation subsided (data not shown). We therefore determined that the inflammation-induced lymphangiogenesis process (subsided) differs from inflammation-induced hemangiogenesis (matured). At this point in the study, there was no evidence of post-transplant inflammation. These observations suggest that the mechanisms underlying the formation of lymphatic vessels are very different from those involved in hemangiogenesis during corneal inflammation, wherein the new blood vessels originate from the limbal circulation, and that the noninflamed cornea contains cells that are capable of participating in the formation of lymphatic vessels during inflammation.

Contribution of adaptive immune cells to bemangiogenesis and lymphangiogenesis after PKP. The rejection reaction after PKP is associated with an antigen-specific, delayed-type hypersensitivity response. Therefore, we examined whether adaptive immune cells (i.e., $T$ and B lymphocytes) play a role in hemangiogenesis and/or lymphangiogenesis after PKP (Figure 2, A-E). We sensitized BALB/c mice to $\mathrm{C} 57 \mathrm{BL} / 6$ alloantigens and compared the corneal graft-induced lymphangiogenesis in immunized versus immunoincompetent (SCID) mice. The levels of hemangiogenesis and lymphangio- 

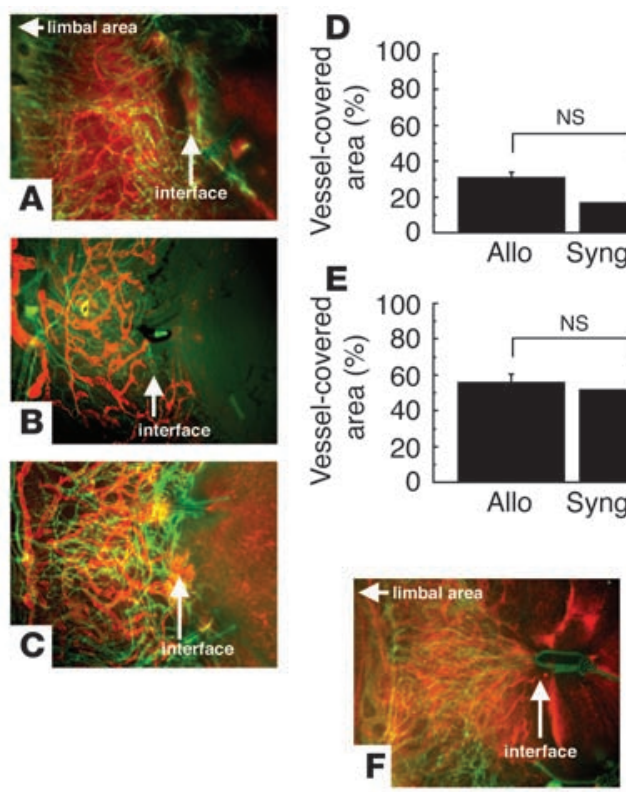

\section{Figure 2}

Comparison of hemangiogenesis and lymphangiogenesis after PKP. (A-C) Photomicrographs of immunofluorescence images 7 days after PKP. (A) Allogeneic (Allo) transplantation. (B) Allogeneic transplantation in SCID mice. (C) Syngeneic transplantation. (D and E) Spot image analysis of lymphangiogenesis visualized by LYVE-1 staining (red) (D) and hemangiogenesis (green) (E) detected by PECAM-1 staining in each transplantation group. (F) Allogeneic transplantation in presensitized hosts. ( $\mathbf{G}$ and $\mathbf{H}$ ) Spot image analysis of lymphangiogenesis $(\mathbf{G})$ and hemangiogenesis $(\mathbf{H})$ in each transplantation group. Magnification, $\times 100$.

genesis did not differ significantly between sensitized and SCID mice on day 3 (data not shown) or even day 7 after transplantation (Figure 2, F-H). These findings indicate that after PKP, hemangiogenesis and lymphangiogenesis are not mediated by an adaptive immune response involving $\mathrm{T}$ or B lymphocytes. Thus, we concluded that hemangiogenesis and lymphangiogenesis were induced by innate immune cells during acute inflammation, as is seen following corneal grafting.

Corneal lymphatic endothelium express CD11b but not CD11c. After ruling out a role for antigen-specific lymphocytes, we examined the ability of innate immune cells to establish lymphatic vessels. Both lymphatic endothelial cells and some of the cells within and around lymphatic vessels expressed LYVE-1. Morphologically, these cells resembled dendritic cells. Immunostaining for the presence of CD11b, CD11c, and LYVE-1 revealed that CD11b and LYVE- $1^{+}$cells, but not CD $11 \mathrm{c}^{+}$cells, contributed to the formation of the lymphatic vessels (Figure 3, A-F). Moreover, 3 days after suture placement, cells positive for both CD11b and Prox-1 were observed in the corneal stroma (Figure 3, G-I). As observed in the central cornea, the presence of $\mathrm{CD} 11 \mathrm{~b}^{+}$cells in the new vessel structures was not continuous with those lymphatic vessels originating from the limbal area (Figure 3, J-M). These observations support the notion that $\mathrm{CD} 11 \mathrm{~b}^{+}$cells, but not $\mathrm{CD} 11 \mathrm{c}^{+}$ cells, are involved in the formation of the pathologic lymphatic vessels and are consistent with the macrophagic origin of these lymphatic endothelial cells.

Presence of bone marrow-derived cells in the walls of lymphatic vessels. Since it has been reported that $\mathrm{CD} 11 \mathrm{~b}^{+}$cells in the cornea are derived from the bone marrow (25), we postulated that bone marrow-derived cells were involved in the assembly of the lymphatic vessels. To assess the role of bone marrow-derived cells in lymphangiogenesis, we adoptively transferred enhanced green fluorescent protein-positive $\left(\mathrm{EGFP}^{+}\right)$bone marrow-derived cells to lethally irradiated mice and determined whether the transcribed cells integrated into newly formed inflammation-induced lymphatic vessels. Three sutures were placed on the cornea of $\mathrm{EGFP}^{+}$chimera mice that had received $\mathrm{EGFP}^{+}$bone marrow cells 2 months earlier. Seven days after suturing, the cornea was removed and stained for LYVE-1. Cell aggregates containing LYVE-1 and EGFP double-positive cells were identified in the cornea (Figure 3, $\mathrm{N}-\mathrm{T})$. Though the transplanted $\mathrm{EGFP}^{+}$cells seemed to be intimately involved with the lymphatic vessel wall, we cannot rule out a role for the resident macrophages since it is possible that cells in tissues may survive after irradiation (26).

Effect of macrophage depletion on lymphangiogenesis. It has been previously shown that local depletion of macrophages by administration of clodronate liposomes suppresses corneal lymphangiogenesis induced by suture placement (4). Similarly, we observed that systemic elimination of macrophages using clodronate liposomes was also effective in suppressing both corneal lymphangiogenesis

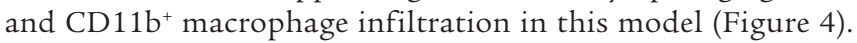
From these results, we concluded that macrophages have an important role in the induction of lymphangiogenesis.

Contribution of blood vascular endothelial progenitor cells to lymphangiogenesis. Previous studies have demonstrated that the circulating blood vascular endothelial progenitor cells (BVEPCs) contribute to corneal hemangiogenesis (27). During the induction of corneal inflammation, BVEPCs are found in the new blood 

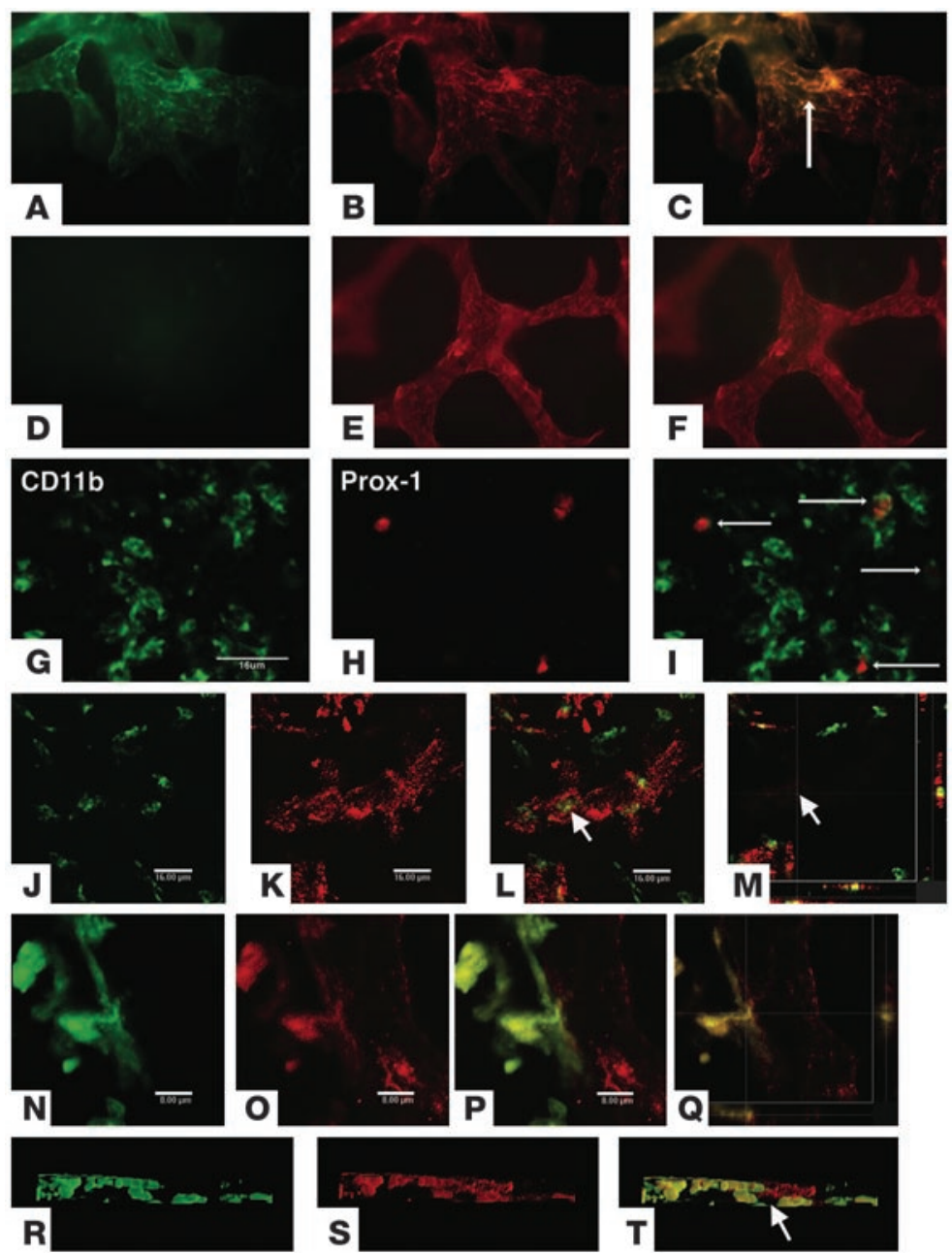

\section{Figure 3}

Lymphatic vascular endothelium after PKP and suture placement. (A-F) Fluorescence microscopy. FITC-conjugated CD11b (A) and Cy3-visualized LYVE-1 (B) staining. (C) Overlay of $\mathbf{A}$ and $\mathbf{B}$ showing dual expression of markers. FITC-conjugated CD11c (D) and Cy3-visualized LYVE-1 (E) staining. (F) Overlay of $\mathbf{D}$ and $\mathbf{E}$ showing dual expression. (G-T) Confocal microscopy. FITC-conjugated CD11b (G) and Cy3-visualized Prox-1 (H) staining. (I) Overlay of G and $\mathbf{H}$ showing dual expression. FITC-conjugated CD11b (J) and Cy3-visualized Prox-1 (K) staining. (L) Overlay of $\mathbf{G}$ and $\mathbf{K}$ showing dual expression. (M) Horizontal section image (bottom) and coronary section image (right) of the lymphatic vessel. (N-Q) EGFP bone marrow cells (green) appear to be part of the lymphatic endothelium or wall. EGFP expression $(\mathrm{N})$ and Cy3-visualized LYVE-1 (O) staining. (P) Overlay of $\mathbf{N}$ and $\mathbf{O}$ showing dual expression. (Q) Horizontal section image (bottom) and coronary section image (right) of the lymphatic wall. (R-T) Three-dimensional image of a lymphatic vessel. EGFP expression (R) and Cy3-visualized LYVE-1 (S) staining. (T) Overlay of $\mathbf{P}$ and $\mathbf{R}$ showing dual expression. Arrows indicate lymphatic vessel lumen. Magnification, $\times 100(\mathbf{A}-\mathbf{F})$. Scale bars: $16 \mu \mathrm{m}(\mathbf{G}-\mathbf{M}) ; 8 \mu \mathrm{m}(\mathbf{N}-\mathbf{Q})$. vessels that arise from the limbus area (27). Endothelial progenitor cells (EPCs) often appear under hypoxic or inflammatory conditions and may share a precursor cell with APCs since they express both CD11b and CD11c (28). We examined whether the lymphatic EPC we observed in our model was a CD11 $\mathrm{b}^{+}$macrophage. For these studies we used C57BL/6 Tie2-LacZ mice in which the Lac $Z$ reporter gene is regulated by the endothelium-specific Tie 2 promoter. Inflammation was induced in the corneas of Tie2-LacZ mice and bone marrow chimera mice with 3 corneal sutures. Seven days later, the corneas were removed, stained for $\beta$-gal and LYVE-1, and examined by confocal microscopy. No $\beta$-gal and LYVE-1 double-positive cells were detected in the lymphatic vessel walls of Tie2-LacZ mice (Figure 5, A-F). Even in the chimeric mice, $\beta$-gal and LYVE- 1 double-positive cells were not detected in the newly formed lymphatic vessel at the same corneal layer (Figure 5, G-L). Similar results were obtained using a Tie2-GFP mouse (data not shown). Thus, we conclude that BVEPCs do not play a role in the formation of the lymphatic vessel wall in the cornea.

Ability of $\mathrm{CD} 11 b^{+}$macrophages to form lymphatic-like tubes. Our in vivo experiments indicated that both bone marrow-derived cells and $\mathrm{CD}_{11} \mathrm{~b}^{+}$macrophages play a role in the formation of pathological lymphatic vessels. Therefore, we performed in vitro studies to investigate whether $\mathrm{CD} 11 \mathrm{~b}^{+}$macrophages were able to form lym- phatic vessel-like structures. CD $11 \mathrm{~b}^{+}$macrophages were collected from the peritoneal cavity of mice using thioglycollate and cultured in RPMI-1640. After 24 hours, the adherent cells were harvested and characterized by flow cytometry. Results revealed that more than $99.67 \%$ of peritoneal exudates cells (PECs) were positive for CD11b (Figure 6A). By RT-PCR, we observed that thioglycollatestimulated $C D 11 b^{+}$macrophages expressed VEGFR-3 and VEGF-C, but not VEGFR-2 (Figure 6B). Of the CD11b+ cells, 30-50\% were also positive for LYVE-1, podoplanin, or Prox-1 (Figure 6C). Immunocytochemical analysis revealed that the PECs (cultured for 24 hours) expressed CD11b and Prox-1 (Figure 6, D-F) as well as podoplanin (Figure 6, G-I) and LYVE-1 protein (Figure 6, J-L). Resting macrophages collected without thioglycollate stimulation showed weak staining for lymphatic endothelial markers compared to thioglycollate-stimulated cells (data not shown). From these results, we concluded that activated, but not resting, $\mathrm{CD} 11 \mathrm{~b}^{+}$macrophages display markers of lymphatic vessels.

In vitro expression of lymphatic endothelial markers by $\mathrm{CD}_{11 b^{+}}$macrophages. We next investigated whether thioglycollate-stimulated $\mathrm{CD}_{1} 1 \mathrm{~b}^{+}$macrophages could form tube-like structures and express lymphatic endothelial markers. CD $11 b^{+}$cells assembled into vessel-like structures, and the ability of the cells to form vessel-like structures varied with cell density. At $10^{5}$ cells $/ \mathrm{ml}, \mathrm{CD} 11 \mathrm{~b}^{+}$ macrophages appeared to become aligned with one another and 

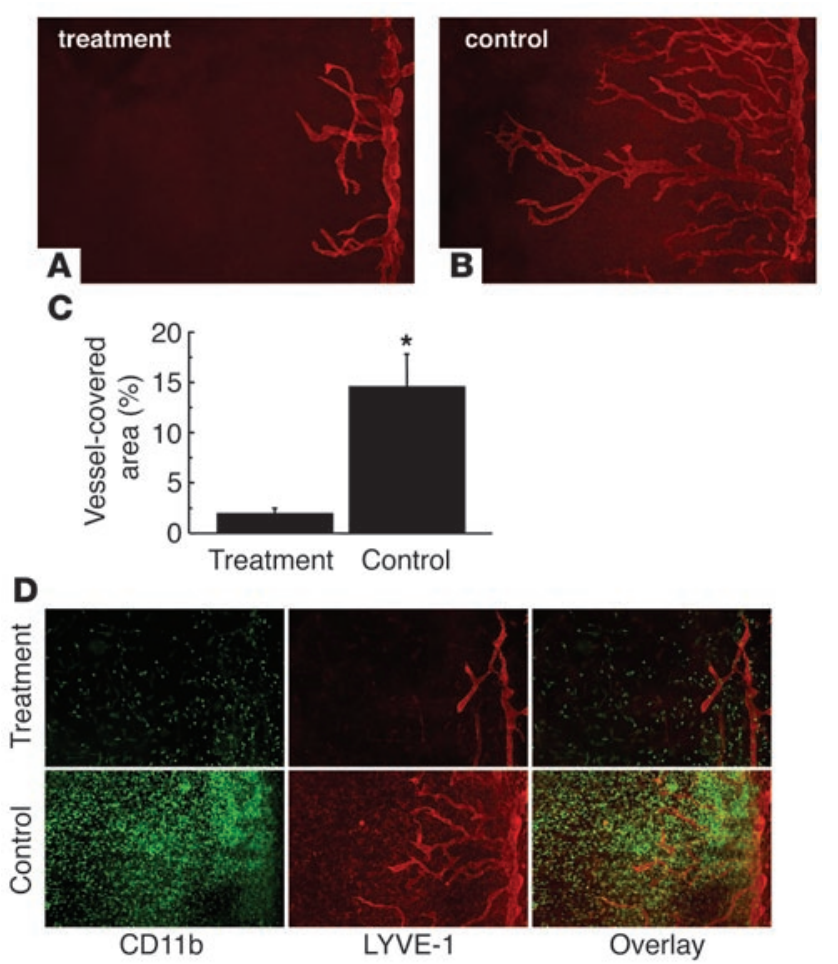

formed a vascular-like network at around day 1 (Figure 7A). At $10^{6}$ cells $/ \mathrm{ml}, \mathrm{CD} 11 \mathrm{~b}^{+}$macrophages associated in clumps between days 1 and 2 and then formed tube-like structures (Figure 7B). Some clumps formed into tube-like structures earlier. We counted both clumps (containing a minimum of 100 cells) and tubelike structures (Figure 7C). At $2 \times 10^{6}$ cells $/ \mathrm{ml}$, the CD $11 \mathrm{~b}^{+}$macrophages survived for at least 31 days, and nearly all the cells were associated with vessel-like structures (Figure 7, D and E). Some of the cells assembled into a structure reminiscent of an oil drum (Figure 7, F-J). The tube-like structures (Figure 7, K, $\mathrm{M}$, and $\mathrm{O}$ ) stained positively for lymphatic endothelial markers podoplanin (Figure 7, L and N) and LYVE-1 (Figure 7, P and R). These observations suggest that $\mathrm{CD}_{11} \mathrm{~b}^{+}$macrophages have the ability to assemble into tube-like structures in vitro.

Transmission electron microscope analysis for the tube-like structure in the Matrigel assay. To evaluate the ultrastructure of the tubes formed by $\mathrm{CD} 11 \mathrm{~b}^{+}$macrophages, the tube-like structures were investigated by transmission electron microscopy. Seven-day Matrigel cultures were prepared as described in Methods and examined by transmission electron microscope (TEM; Figure 8, $\mathrm{A}$ and B). The tube-like structures had lumens (Figure 8C), and their walls were composed of loosely associated macrophages lacking continuous basal lamina. The margins of the endothelial cells appeared to be joined to adjacent cells (Figure 8, D and E) by interdigitating or overlapping contacts (Figure 8, F and G) and resembled filament-like tentacles.

\section{Discussion}

We show here that monocytic cells - especially CD $11 \mathrm{~b}^{+} \mathrm{CD}^{-11 \mathrm{c}^{-}}$ macrophages, but not $\mathrm{CD}_{11} \mathrm{~b}^{-} \mathrm{CD} 11 \mathrm{c}^{+}$dendritic cells or BVEPCs - contributed to the formation of new lymphatic vessels in the inflamed cornea and had the ability to form lymphatic vessel-like structures in vitro. The lymphatic nature of the cells was demon-

\section{Figure 4}

Effect of macrophage depletion on lymphangiogenesis after suture placement. (A and B) Spot image analysis of lymphangiogenesis visualized by LYVE-1 staining (red) in each group. (A) Clodoronate liposomes (treatment); (B) PBS-containing liposomes (control). (C) Quantification of lymphatic vessel invasion 7 days after suture placement $(n=5)$. (D) Immunofluorescence images showing CD11b (green) and LYVE-1 (red) staining 7 days after suture placement in mice treated with clodoronate liposomes or PBS-containing liposomes. ${ }^{*} P<0.0005$. Magnification, $\times 100$.

strated by their expression of not only LYVE-1 but also Prox-1 and podoplanin. Prox-1 is a key regulator in the molecular pathway that forms the lymphatic vasculature and has been identified as a master switch in the mechanism specifying lymphatic endothelial cell fate (29). Moreover, systematic elimination of macrophages using clodronate liposomes led to a near-complete inhibition of lymphatic vessel formation in the inflamed cornea.

We were therefore interested in determining whether the lymphatic vessels in the inflamed cornea in vivo were formed from indigenous or recruited cells. We used EGFP chimeric mice to test the possibility that bone marrow-derived cells were responsible for the generation of new lymphatic vessels. Our results demonstrated the presence of EGFP-labeled cells in discrete patches in the newly formed corneal lymphatic vessel wall, indicating that bone marrow-derived cells were involved in the establishment of the lymphatic vasculature following sutureinduced inflammation. Another possible explanation was that bone marrow-derived BVEPCs were contributing to the formation of lymphatic vessels. However, we did not detect any Tie2positive cells in the nascent lymphatic vessels following transplantation of bone marrow cells from Tie2-LacZ (or Tie2-GFP) mice. Therefore BVEPCs, which are well documented for their role in angiogenesis, do not appear to have a role in the development of lymphatic vasculature in the inflamed cornea. The observation that BVEPCs did not contribute to the formation of corneal lymphatics is consistent with previous reports demonstrating that endothelial cells sprouting from new blood vessels do not undergo lymphatic differentiation (16).

Since we had ruled out a role for adaptive immunity in the development of inflammation-mediated lymphangiogenesis, we postulated that innate immune cells and their responses triggered corneal hemangiogenesis and lymphangiogenesis immediately following PKP. LYVE-1 ${ }^{+}$cells clearly exhibited a macrophage/dendritic cell morphology. This conclusion was supported by a previous report showing that lymphangiogenesis is eliminated in vivo by local treatment with clodronate liposomes (4) and our present observation that when systemic administration of clodronate liposomes depleted $\mathrm{CD} 11 \mathrm{~b}^{+}$macrophages, infiltration and formation of lymphatic vessels were suppressed. It is known that the clodronate liposomes are taken up selectively by phagocytic cells, which indicates that dendritic cells do not contribute to the lymphatic endothelium (30-32). Thus, we concluded that CD11 $\mathrm{b}^{+}$macrophages have an important role in the induction of lymphangiogenesis in the inflamed cornea.

Our studies also revealed a mechanism for the development of corneal lymphatic vessels that differs from previously known mechanisms of corneal hemangiogenesis or postnatal lymphangiogenesis in any tissue to our knowledge. It is known that during hemangiogenesis, the vessels sprout from preexisting limbal 


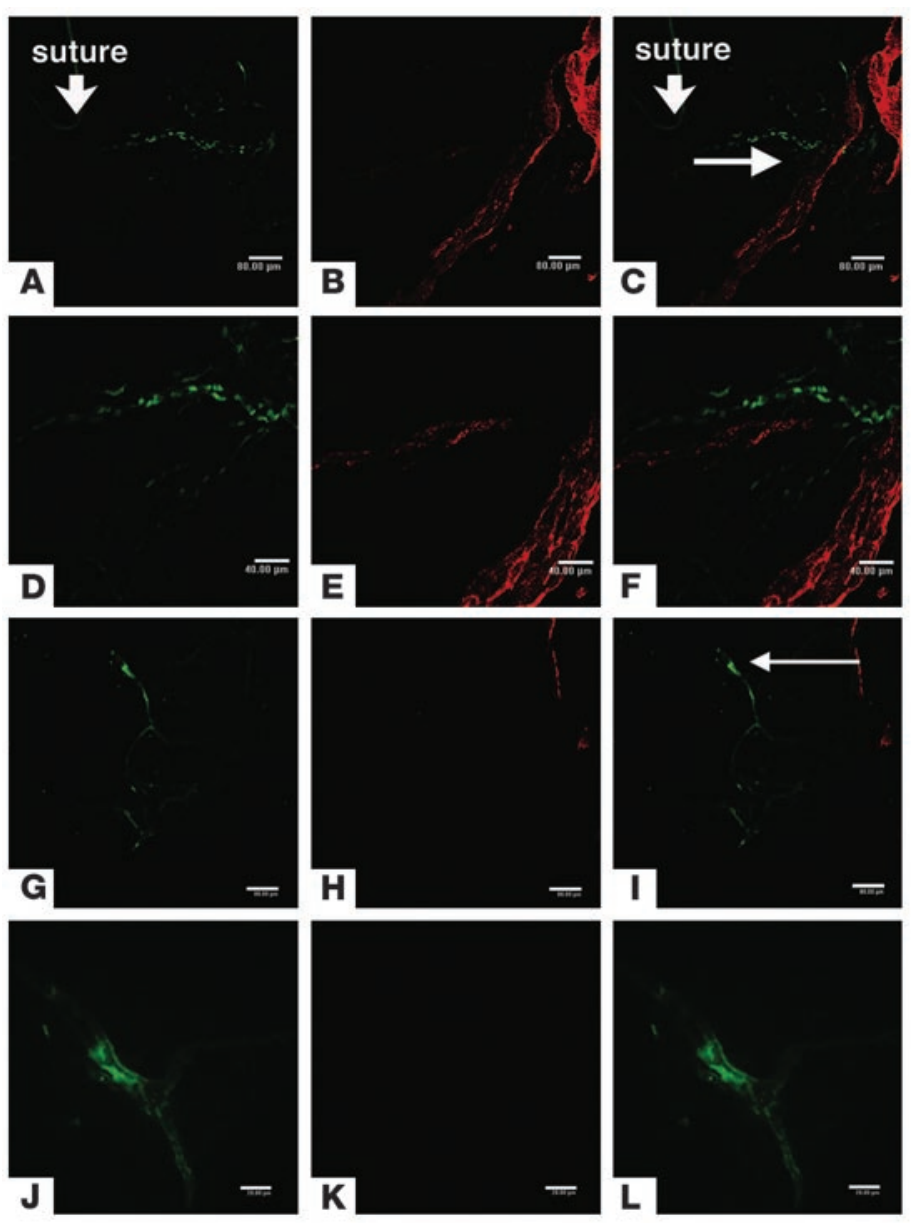

\section{Figure 5}

Involvement of EPCs in lymphatic vascular development following corneal suture. (A-C) Tie2-LacZ mouse cornea. Cy2-visualized $\beta$-gal (A) and Cy3-visualized LYVE-1 (B) staining. (C) Overlay of $\mathbf{A}$ and $\mathbf{B}$ showing dual expression. (D-F) Higher magnification view of area indicated by arrows in $\mathbf{A}$ and $\mathbf{C}$. Cy2-visualized $\beta$-gal (D) and Cy3-visualized LYVE-1 (E) staining. (F) Overlay of $\mathbf{E}$ and $\mathbf{F}$ showing dual expression. (G-I) LacZ expression at the blood vessel, not lymphatic in Tie2-LacZ chimera mouse cornea. Cy2-visualized $\beta$-gal (G) and Cy3-visualized LYVE-1 (H) staining. (I) Overlay of $\mathbf{G}$ and $\mathbf{H}$ showing dual expression. (J-L) Higher magnification view of area indicated by arrow in I. Cy2-visualized $\beta$-gal (J) and Cy3-visualized LYVE-1 (K) staining. (L) Overlay of $\mathbf{J}$ and $\mathbf{K}$ showing dual expression. Scale bars: $80 \mu \mathrm{m}$ (A-C and G-I), $40 \mu \mathrm{m}$ (D-F), and $20 \mu \mathrm{m}$ (J-L).

vessels (4). Here we observed that during corneal inflammation, some of the lymphatic vessels appeared to arise de novo within the corneal stroma at a distance from the limbus, with no apparent connection to the limbal lymphatic structures. These nascent lymphatic structures then connected to the limbal lymphatic vessels. Interestingly, this phenomenon appeared to be recapitulated in an in vitro tube-formation assay wherein highly enriched thioglycollate-activated CD $11 \mathrm{~b}^{+}$macrophages cultured in Matrigel initially formed aggregates before forming tube-like structures. These vessel-like structures expressed lymphatic endothelial markers, including LYVE-1, Prox-1, and podoplanin. Additionally, results of ultrastructural analysis of tubes formed in vitro reveal that, like our lymphatic vessels in vivo, the structures lack a continuous basal lamina (33). Thus, $\mathrm{CD}_{1} 1 \mathrm{~b}^{+}$macrophages form tube-like structures, which express both the lymphatic endothelial markers and architecture of lymphatic vessels in situ.

It has been shown that VEGF-C participates in the development of the lymphatic vasculature through activation of VEGFR-3. VEGF-C also plays a role in lymphangiogenesis in tumor development and inflammatory conditions (34). Consistent with these data, we previously observed that $\mathrm{CD} 11 \mathrm{~b}^{+}$macrophages within the inflamed corneal stroma express VEGF-C, and that bone marrow-derived macrophages express both $V e g f c$ and $V e g f d$ mRNA (4). However, lymphangiogenesis may be induced under pathological conditions by not only VEGF-C stimulation, but by other inflam- matory mediators such as IL-1 $\beta$, LPS, and PDGF-BB as well (35). Our present in vitro data showed that exogenous VEGF-C stimulation did not accelerate the formation of clusters and tube-like structures, nor did it affect LYVE-1 expression (as measured by flow cytometric analysis; data not shown). We suspect that the exogenous addition of VEGF-C had no effect because the activated macrophages already secrete sufficient VEGF-C to induce maximal phenotypic effect. In addition, we speculate that tube formation in the Matrigel assay was density dependent simply because a threshold number of cells is needed to form the structures. This hypothesis is supported by our in vivo observation that $\mathrm{CD} 11 \mathrm{~b}^{+}$macrophages in the control corneal stroma, which were few in number, formed many fewer lymphatic structures than after suture placement, when the number of macrophages increased dramatically (Figure 4).

This report provides strong evidence that a subpopulation of $\mathrm{CD}_{11} \mathrm{~b}^{+}$macrophages has lymphatic endothelial characteristics and may be involved in the induction of pathological lymphangiogenesis. Moreover, the critical role for inflammation in corneal lymphangiogenesis raises significant questions about the role of inflammatory macrophages in the development of lymphatic vessels in other tissues and organs. While we cannot rule out the possibility that a rare cell type or a cell with an unusual pattern of marker expression exists in the population of cells that produce lymphatic vessels, we feel that therapeutic prevention of macrophage activation might prove to successfully prolong cor- 
A

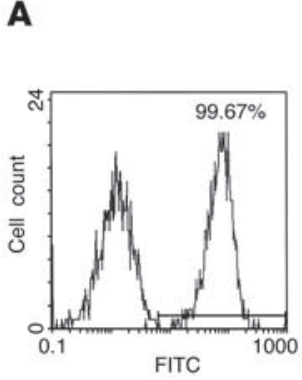

B
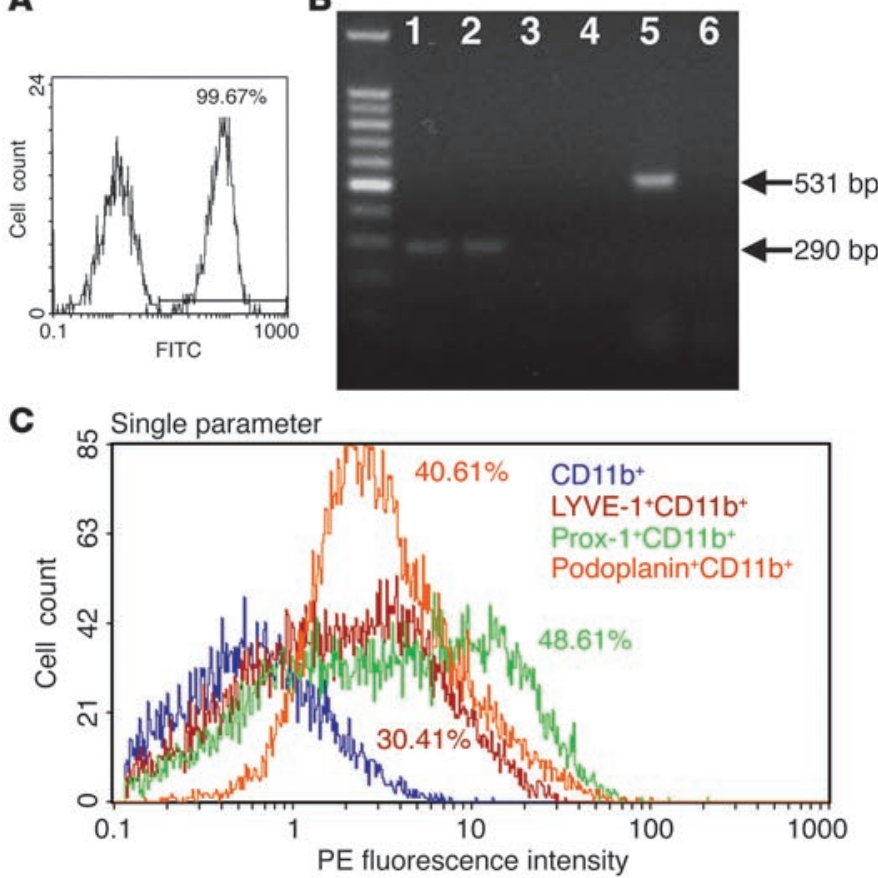
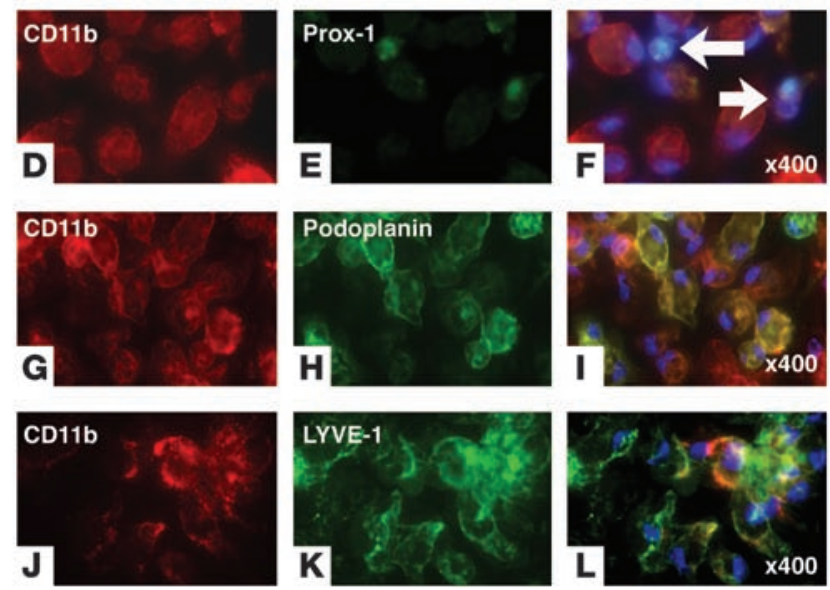

\section{Figure 6}

Characterization of PECs. (A) Flow cytometric analysis of thioglycollate-activated adherent PECs. Percentage of PECs positive for CD11b is shown. The $x$ axis shows FITC fluorescence intensity. (B) RT-PCR of CD11 b+ macrophages. Shown are CD11b macrophages stimulated with thioglycollate VEGFR-3 (lane 1), VEGFR-2 (lane 3), and VEGF-C (lane 5) and their unstimulated counterparts (lanes 2, 4, and 6, respectively). The far left lane provides size markers. (C) Multicolor FACS analysis of CD11 b+ macrophages. Percentages of double-positive cells are shown. (D-L) Immunocytochemistry of thioglycollate-activated adherent PECs. PE-conjugated CD11b (D) and FITC-visualized Prox-1 (E) staining. (F) Overlay of D and $\mathbf{E}$ showing dual expression. PE-conjugated CD11b (G) and FITC-visualized podoplanin (H) staining. (I) Overlay of $\mathbf{G}$ and $\mathbf{H}$ showing dual expression. PE-conjugated CD11b (J) and FITC-visualized LYVE-1 (K) staining; (L) overlay of $\mathbf{J}$ and $\mathbf{K}$ showing dual expression. Magnification, $\times 400$.

neal graft survival. It is possible that macrophages surrounding tumors and other sites of inflammation may be involved in the orchestration of lymphatic vessels; therefore, understanding this novel mechanism for lymphangiogenesis within the cornea may have important ramifications for antilymphangiogenesis therapies in the treatment of a variety of diseases.

\section{Methods}

Animals. For corneal transplant experiments, male BALB/c and CB17 SCID mice (aged 8-10 weeks) were used as hosts, and age-matched male $\mathrm{BALB} / \mathrm{c}$ and $\mathrm{C} 57 \mathrm{BL} / 6$ mice were the donors (Taconic). Male BALB/c mice (Taconic) were used in the suture-placement model. All animal protocols were approved by the Schepens Eye Research Institute Animal Care and Use Committee in accordance with NIH guidelines and the Association for Research in Vision and Ophthalmology's Statement for the Use of Animals in Ophthalmologic and Vision Research.

Mouse corneal transplantation and corneal suture placement. Prior to PRK, each animal was deeply anesthetized with an intraperitoneal injection of ketamine $(3 \mathrm{mg})$ and xylazine $(0.007 \mathrm{mg})$ before the central $2-\mathrm{mm}$ portion of the donor cornea was excised with vannas scissors and secured in recipient graft beds with 8 interrupted 11-0 nylon sutures (36). Using stromal incursions that encompassed more than $120^{\circ}$ of the corneal circumference, 3 11-0 nylon sutures were placed intrastromally. To obtain a standardized angiogenic response, the outer edge of the suture was placed halfway between the limbus and the line outlined by the 2 -mm trephine; the inner edge was equidistant from the 2-mm trephine. Sutures were left in place for 7 days.
Whole-mount corneal staining and morphological determination of hemangiogenesis and lymphangiogenesis. Mice were euthanized on day 3 or 7 after PKP. The corneas were excised, rinsed 3 times in PBS, and fixed in acetone for 1 hour. They were then rinsed once in PBS, blocked with $2 \%$ BSA-PBS, incubated overnight at $4^{\circ} \mathrm{C}$ with rabbit anti-mouse LYVE-1 antibody (1:500; a kind gift of D.G. Jackson, Oxford University, Oxford, United Kingdom) $(8,37)$ and rabbit anti-mouse Prox-1 antibody (1:500; Upstate). The tissues were then washed, blocked, and stained overnight with FITC-conjugated CD11b (1:100; BD Biosciences - Pharmingen), purified CD11c (1:100; BD Biosciences - Pharmingen), and FITC-conjugated CD31 (PECAM-1, 1:100; Santa Cruz Biotechnology Inc.) antibodies and finally washed, blocked, and stained with Cy3-conjugated secondary antibody (1:100; Jackson ImmunoResearch Laboratories). For isotype control of CD11b, rat (DA) $\operatorname{IgG}_{2 b}, \mathrm{~K}$ (BD Biosciences - Pharmingen) was used. Double-stained whole-mount sections were analyzed under a Zeiss Axiophot microscope and a Leica TSC-SP2 inverted and upright confocal laser-scanning microscope. Digital pictures of the flat mounts were taken using a spot image analysis system (SPOT Advanced version 3.2, Diagnostic Instruments Inc.), and the area covered by blood and lymphatic vessels positive for CD31/LYVE-1 $(8,37)$ was measured using NIH ImageJ version 1.33u (http://rsb.info.nih.gov/ij/). The total corneal area was outlined using the innermost vessel of the limbal arcade as the border; the area of blood and lymphatic neovascularization within the cornea was calculated and normalized to the total corneal area (expressed as the percentage of the cornea covered by vessels).

Corneal explant cultures. An area $2 \mathrm{~mm}$ in diameter was excised from the mouse central cornea, rinsed 3 times with PBS, placed epithelium side-up 

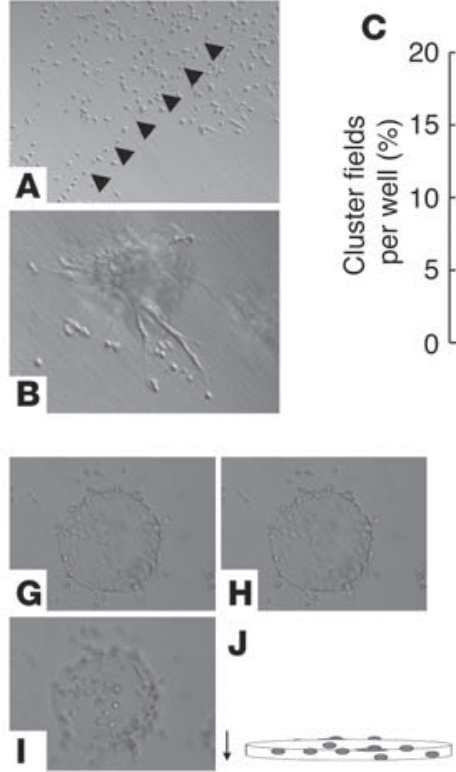
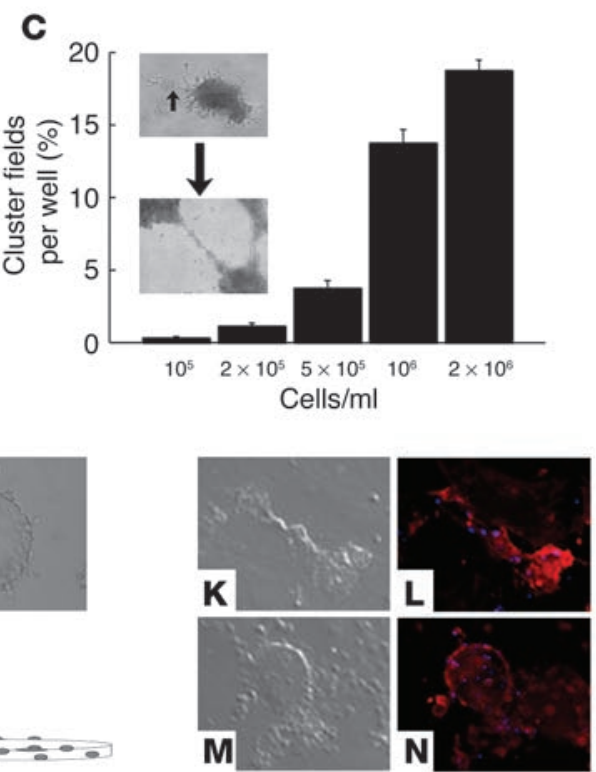
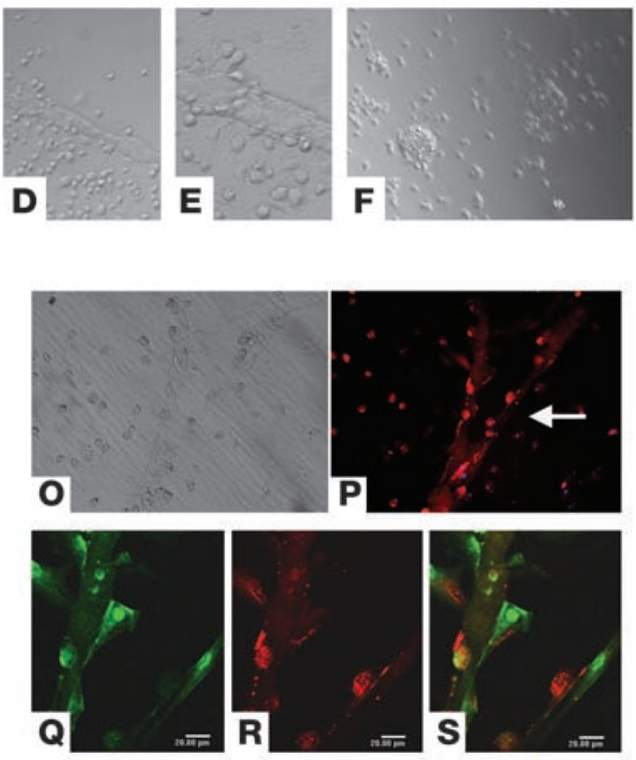

\section{Figure 7}

Behavior of PEC in a tube-formation assay. (A and B) Matrigel assay at day 3 containing $10^{5} \mathrm{cells} / \mathrm{ml}$. (A) PECs appear to be aligning end-to-end in the assay (arrowheads). (B) PECs associating to form a vessel-like structure. (C) Measurement of aggregation and tube-like structure field in the Matrigel assay at day 2. (D-S) Long-term (31 days) assay containing $2 \times 10^{6} \mathrm{cells} / \mathrm{ml}$. (D and E) Micrograph of the tube-like structure in the Matrigel (31 days). (F-H) Another area of a Matrigel assay (day 31) containing $2 \times 10^{6} \mathrm{cells} / \mathrm{ml}$. Shown are sequential images of upper (G), middle $(\mathbf{H})$, and lower (I) levels of matrigel cultures. (J) Horizontal view of images in $\mathbf{G}-\mathbf{I}$. (K, M, and $\mathbf{O})$ Matrigel assay at day 31 containing $2 \times 10^{6}$ cells/ml. (L and N) Cy3-visualized podoplanin staining and nuclear staining with DAPI (blue). (P) Fluorescence microscopy image of matrigel cultures. Arrow depicts area evaluated by confocal microscopy in $\mathbf{Q}-\mathbf{S}$. (Q-S) Confocal image of Matrigel at day 31 . EGFP expression (Q) and Cy3-visualized LYVE-1 staining and nuclear staining with DAPI (P and $\mathbf{R})$. (S) Overlay of $\mathbf{Q}$ and $\mathbf{R}$ showing dual expression. Magnification, $\times 100$ (A, C, and F); ×200 (B, D, and K-P); ×400 (E and G-I). Scale bars in Q-S: $20 \mu \mathrm{m}$.

in the well of 96-well dishes containing 10\% BSA (Sigma-Aldrich), $20 \mathrm{ng}$ IL-1 $\beta$ (BD Biosciences - Pharmingen), and $100 \mathrm{U} / \mathrm{ml}$ penicillin and $100 \mu \mathrm{g}$ streptomycin (Biowhitaker) in DMEM medium (Biowhitaker), and incubated for $48-72$ hours at $37^{\circ} \mathrm{C}$ in a $5 \% \mathrm{CO}_{2}$ atmosphere. The cornea was stained with LYVE-1 and CD31 using the method described for wholemount corneal staining.

Irradiation and bone marrow transplantation. Male C57BL/6 mice, aged 7-8 weeks, were transplanted with bone marrow from transgenic mice expressing various promoters, including EGFP and Tie2-LacZ (38).

Bone marrow cells were obtained by flushing the tibias and femurs of age-matched donor EGFP transgenic mice (C57BL/6-TgN [ACTbEGFP] 10sb; The Jackson Laboratory) and Tie2-LacZ transgenic mice (C57BL/6-TgN [TIE2LacZ] 182Sato; The Jackson Laboratory). Low-density mononuclear bone marrow cells were isolated by density gradient centrifugation over Histopaque-1083 (Sigma-Aldrich). Eight weeks after bone marrow transplantation, when the bone marrow of the recipients was considered to be completely reconstituted (27), the mice underwent corneal suturing.

Detection of bone marrow cells positive for EGFP and double-positive for LYVE-1 and $\beta$-gal expression in corneal tissue. The mice were euthanized, and their corneas were excised along the limbus area. A modified flatmount double immunohistochemistry technique was used in which corneal flat mounts were fixed in $4 \%$ paraformaldehyde and then blocked and stained with LYVE-1 $(1: 500)(8,37)$ visualized with Cy3conjugated secondary antibody $(1: 100)$, and blocked and stained with anti-rabbit polyclonal $\beta$-galactosidase antibody (1:300; Jackson ImmunoResearch Laboratories) (39) visualized with Cy2-conjugated secondary antibody (1:100) and protected with Vectorshields (Vector Labora- tories) for fluorescence detection. Tissues were examined with a Leica TSC-SP2 inverted and upright confocal laser scanning microscope and a Zeiss Axiophot microscope.

Systemic depletion of macrophages using clodronate liposomes. Systemic depletion of monocytes/macrophages was accomplished as described previously (40). $\mathrm{Cl}_{2} \mathrm{MDP}$ (clodronate) liposomes, a gift of Roche Diagnostics $\mathrm{GmbH}$, was injected via the tail vein 3 days before suture placement, at the time of suture placement, and 3 and 7 days after surgery. Control mice received liposomes containing PBS at the same time points.

Collection and culture of macrophages from the peritoneal cavity. Thioglycollate-induced macrophages (PECs) were collected from the peritoneal cavity of normal 8-week-old male C57BL/6 and EGFP mice (41). PECs were washed, resuspended, and cultured $\left(10^{6}\right.$ cells/plate, 24 hours at $37^{\circ} \mathrm{C}$ in a $5 \% \mathrm{CO}_{2}$ atmosphere) in $35-\mathrm{mm}$ culture plates in RPMI- 1640 medium containing 10\% BSA (Sigma-Aldrich), $1 \times 10^{-5} \mathrm{M} 2$-ME (mercaptoethanol; Sigma-Aldrich), $10 \mathrm{mM}$ HEPES, $0.1 \mathrm{mM}$ nonessential amino acid, $1 \mathrm{mM}$ sodium pyruvate, $100 \mathrm{U} / \mathrm{ml}$ penicillin, and $100 \mu \mathrm{g} / \mathrm{ml}$ streptomycin (Biowhitaker).

Flow cytometry, immunocytochemistry, and RT-PCR analysis of PECs. Nonadherent cells were removed, and adherent cells were harvested by scraping from the culture dish. The cells were rinsed in PBS, incubated for 10 minutes on ice with CD16/CD32 Fc block (BD Biosciences - Pharmingen) in $2 \%$ bovine serum-PBS, and stained for 30 minutes on ice with FITCconjugated CD11b, nonfluorescence-conjugated LYVE-1, podoplanin (Research Diagnostics Inc.), Prox-1, and isotype controls (FITC-conjugated nonspecific rat IgG2b, PE-conjugated hamster IgG, nonconjugated rabbit IgG; BD Biosciences - Pharmingen). For the LYVE-1, Prox-1, and rabbit isotype controls, $\mathrm{Cy} 3$-conjugated goat anti-rabbit secondary anti- 


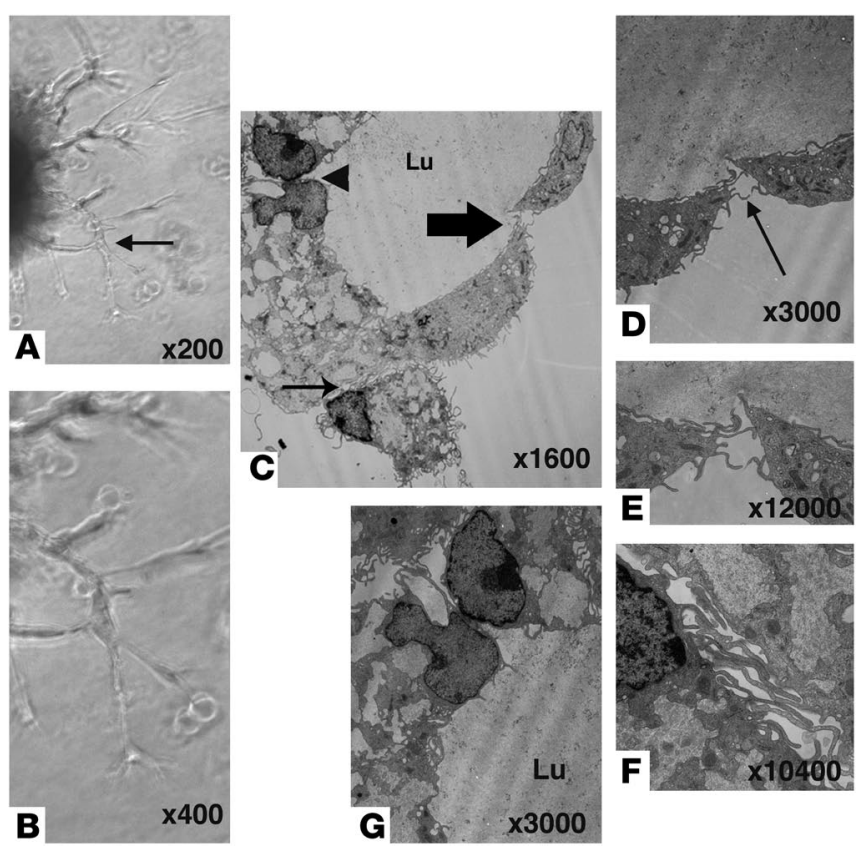

body was used; for podoplanin, Cy3-conjugated goat anti-Syrian hamster secondary antibody was used. Labeled cells were analyzed on an EPIC XL flow cytometer (Beckman Coulter). For multicolor analysis, the analysis gate was only set to detect FITC-CD $11 b^{+}$cells.

For immunocytochemistry, CD $11 \mathrm{~b}^{+}$PECs in the tissue culture dishes were rinsed with PBS, fixed in $4 \%$ paraformaldehyde for 7 minutes, rinsed with PBS, blocked in $2 \%$ BSA containing $0.1 \%$ Triton-X-PBS, stained with LYVE-1 (1:500), podoplanin (1:500), or Prox-1 (1:500), rinsed, and blocked as described above, stained with FITC-conjugated CD11b (1:250) and Cy3-conjugated (1:500) secondary antibodies, washed with PBS, and covered with a Vectorshield with DAPI applied for fluorescence. Analysis was performed under a Zeiss Axiophot microscope. For RT-PCR analysis, total RNA from PECs was extracted using TRIzol (Invitrogen Corp.). First-strand cDNA was prepared from total RNA by reverse transcriptase using oligo (dT) primers. For the detection of VEGF-C, primers expressing VEGFR-3 and VEGFR-2 were used in the RT-PCR (4).

Assay of tube formation using $C D 11 b^{+}$PECs. Only adherent cells that expressed $\mathrm{CD} 11 \mathrm{~b}$ were used in the tube-formation assay. Growth factor-reduced Matrigel (100 $\mu \mathrm{l}$; BD) prepared from an Engelbreth-HolmSwarm tumor was added to 4-chamber slides (Lab-Tek; Nunc) with $100 \mu \mathrm{l}$ endothelial cell medium (EBM-2; Cambrex Corp.) and allowed to gel for 30 minutes at $37^{\circ} \mathrm{C}$. PECs were seeded at densities of $10^{5}, 2 \times 10^{5}, 5 \times 10^{5}, 10^{6}$, and $2 \times 10^{6} \mathrm{cells} / \mathrm{ml}$ in $500 \mu \mathrm{l}$ of EBM- 2 containing $3 \% \mathrm{FBS}$. Tube formation was monitored over 31 days.

Digital pictures were taken using a spot image analysis system, and the area covered by both aggregate and tube-like structures were measured using NIH Image software at 48 hours. The total area covered by the Matrigel was outlined $\left(18 \mathrm{~mm}^{2}\right)$; the area of the aggregate and the tube-like structures within the Matrigel was calculated and expressed as a percentage of the Matrigel area. Exogenous VEGF-C (100 ng/ml; Research Diagnostics Inc.) was added to the gel assay, and 24 hours later the clusters and tubelike structures were assayed as described above.

Detection of LYVE-1 and podoplanin expression in tube-like structures. CD $11 \mathrm{~b}^{+}$ PECs were stained for the presence of LYVE-1 and podoplanin at D7 and D31 after the establishment of Matrigel cultures. CD11 b PECs in Matrigel were rinsed in PBS, fixed in $4 \%$ paraformaldehyde for 7 minutes, rinsed in

\section{Figure 8}

TEM image of a tube-like structure formed by CD11 b+ PECs. (A and B) Three-dimensional visualization of $\mathrm{CD} 11 \mathrm{~b}^{+}$macrophage tube formation (described in Figure 6) at day 7 of Matrigel assay. Panel B shows a higher magnification of the region in $\mathbf{A}$ indicated by the arrow. (C-G) Tube-like structure in Matrigel assay. (C) Lumen (Lu) formed by CD11 b+ macrophages. Panels D, F, and $\mathbf{G}$ show higher magnification views of the regions in $\mathbf{C}$ indicated by the large arrow, small arrow, and arrowhead, respectively. Panel $\mathbf{E}$ shows a higher magnification view of the region in $\mathbf{D}$ indicated by the arrow. Magnifications are as indicated in each panel.

PBS, blocked in 2\% BSA containing 0.1\% Triton-X-PBS, stained with LYVE-1 (1:500) and podoplanin (1:500), washed, blocked, stained with Cy3-conjugated secondary antibody (1:500), washed with PBS, and covered with Vectorshields for fluorescence detection. Analysis was performed with a Zeiss Axiophot microscope and a Leica TSC-SP2 inverted and upright confocal laser scanning microscope.

Transmission electron microscopy. The Matrigel samples were fixed with 1:1 Karnovsky fixative (4\% paraformaldehyde, $2.5 \%$ glutaraldehyde) and postfixed in osmium tetroxide. The samples then were dehydrated with $50 \%$, $70 \%, 95 \%$, and $100 \%$ ethanol and propylene oxide prior to embedding in epoxy (embed 812). The grids were stained with lead citrate and uranyl acetate. Samples were cut and observed under a Philips 410 TEM.

Statistical analysis. Statistical significance was determined using the Mann Whitney $U$ test. Differences were considered significant at $P<0.05$. Each experiment was performed at least twice with similar results. Graphs were drawn using StatView version 5.0 (SAS Institute Inc.).

\section{Acknowledgments}

We thank Reza Dana for the many helpful discussions and critical reading of the manuscript. We appreciate our beneficial interactions with Lu Chen. We also thank Jacqueline Doherty for general support, Donald Pottle for help with confocal and immunofluorescent imaging, Marie Ortega for excellent management of the Schepen's vivariam, Patricia Pearson for help with TEM imaging, Diane Darland and Magali Saint-Geniez for discussion of TEM imaging, and Randy Huang for assistance in analysis of flow cytometry. We especially thank Dom Sanethong for administrative assistance in the preparation of this manuscript. This work was supported by NIH grants EY10765 (to J.W. Streilein and J. Stein-Streilein), EY11893 (to J. Stein-Streilein), and EY05318 (to P.A. D’Amore).

Received for publication November 10, 2004, and accepted in revised form June 7, 2005.

Address correspondence to: Joan Stein-Streilein, Ocular Immunology Group, The Schepens Eye Research Institute, Harvard Medical School, 20 Staniford Street, Boston, Massachusetts 02114, USA. Phone: (617) 912-7494; Fax: (617) 912-0105; E-mail: jstein@vision. eri.harvard.edu.

Claus Cursiefen's current address is: Department of Ophthalmology, Universität Erlangen-Nürnberg, Erlangen, Germany.

Kazuichi Maruyama, Masaaki Ii, and Claus Cursiefen contributed equally to this work.

J. Wayne Streilein is deceased. 
1. Cursiefen, C., Chen, L., Dana, M.R., and Streilein, J.W. 2003. Corneal lymphangiogenesis: evidence, mechanisms, and implications for corneal transplant immunology [review]. Cornea. 22:273-281.

2. Streilein, J.W., Yamada, J., Dana, M.R., and Ksander, B.R. 1999. Anterior chamber-associated immune deviation, ocular immune privilege, and orthotopic corneal allografts. Transplant. Proc. 31:1472-1475.

3. Dana, M.R., and Streilein, J.W. 1996. Loss and restoration of immune privilege in eyes with corneal neovascularization. Invest. Ophthalmol. Vis. Sci. 37:2485-2494.

4. Cursiefen, C., et al. 2004. VEGF-A stimulates lymphangiogenesis and hemangiogenesis in inflammatory neovascularization via macrophage recruitment. J. Clin. Invest. 113:1040-1050. doi:10.1172/JCI200420465.

5. Jackson, D.G. 2001. New molecular markers for the study of tumour lymphangiogenesis. Anticancer Res. 21:4279-4283.

6. Casley-Smith, J.R. 1980. The fine structure and functioning of tissue channels and lymphatics. Lymphology. 13:177-183.

7. Leak, L.V. 1970. Electron microscopic observations on lymphatic capillaries and the structural components of the connective tissue-lymph interface. Microvasc. Res. 2:361-391.

8. Cursiefen, C., et al. 2002. Lymphatic vessels in vascularized human corneas: immunohistochemical investigation using LYVE-1 and podoplanin. Invest. Ophthalmol. Vis. Sci. 43:2127-2135.

9. Skobe, M., et al. 2001. Induction of tumor lymphangiogenesis by VEGF-C promotes breast cancer metastasis. Nat. Med. 7:192-198.

10. Breiteneder-Geleff, S., et al. 1997. Podoplanin, novel 43-kd membrane protein of glomerular epithelial cells, is down-regulated in puromycin nephrosis. Am. J. Pathol. 151:1141-1152.

11. Breiteneder-Geleff, S., et al. 1999. Podoplanin--a specific marker for lymphatic endothelium expressed in angiosarcoma [In German]. Verh. Dtsch. Ges. Pathol. 83:270-275.

12. Sinzelle, E., et al. 2000. Intrapericardial lymphangioma with podoplanin immunohistochemical characterization of lymphatic endothelial cells. Histopathology. 37:93-94.

13. Weninger, W., et al. 1999. Expression of vascular endothelial growth factor receptor-3 and podoplanin suggests a lymphatic endothelial cell origin of Kaposi's sarcoma tumor cells. Lab. Invest. 79:243-251.

14. Banerji, S., et al. 1999. LYVE-1, a new homologue of the CD44 glycoprotein, is a lymph-specific receptor for hyaluronan. J. Cell Biol. 144:789-801.
15. Prevo, R., Banerji, S., Ferguson, D.J., Clasper, S., and Jackson, D.G. 2001. Mouse LYVE-1 is an endocytic receptor for hyaluronan in lymphatic endothelium. J. Biol. Chem. 276:19420-19430.

16. Wigle, J.T., et al. 2002. An essential role for Prox1 in the induction of the lymphatic endothelial cell phenotype. EMBO J. 21:1505-1513.

17. Wigle, J.T., Chowdhury, K., Gruss, P., and Oliver, G. 1999. Prox1 function is crucial for mouse lens-fibre elongation. Nat. Genet. 21:318-322.

18. Hamrah, P., Liu, Y., Zhang, Q., and Dana, M.R. 2003. The corneal stroma is endowed with a significant number of resident dendritic cells. Invest. Ophthalmol. Vis. Sci. 44:581-589.

19. Mimura, T., et al. 2001. Expression of vascular endothelial growth factor $\mathrm{C}$ and vascular endothelial growth factor receptor 3 in corneal lymphangiogenesis. Exp. Eye Res. 72:71-78.

20. Hamrah, P., et al. 2004. Expression of vascular endothelial growth factor receptor-3 (VEGFR-3) on monocytic bone marrow-derived cells in the conjunctiva. Exp. Eye Res. 79:553-561.

21. Skobe, M., et al. 2001. Concurrent induction of lymphangiogenesis, angiogenesis, and macrophage recruitment by vascular endothelial growth factorC in melanoma. Am. J. Pathol. 159:893-903.

22. Schoppmann, S.F., et al. 2002. Tumor-associated macrophages express lymphatic endothelia growth factors and are related to peritumoral lymphangiogenesis. Am. J. Pathol. 161:947-956.

23. Loughman, M.S., et al. 1996. Experimental corneal neovascularisation using sucralfate and basic fibroblast growth factor. Aust. N. Z. J. Ophthalmol. 24:289-295.

24. Gimbrone, M.A., Jr., Leapman, S.B., Cotran, R.S., and Folkman, J. 1973. Tumor angiogenesis: iris neovascularization at a distance from experimental intraocular tumors. J. Natl. Cancer Inst. 50:219-228.

25. Hamrah, P., Huq, S.O., Liu, Y., Zhang, Q., and Dana, M.R. 2003. Corneal immunity is mediated by heterogeneous population of antigen-presenting cells. J. Leukoc. Biol. 74:172-178.

26. Schilling, M., et al. 2003. Microglial activation precedes and predominates over macrophage infiltration in transient focal cerebral ischemia: a study in green fluorescent protein transgenic bone marrow chimeric mice. Exp. Neurol. 183:25-33.

27. Takahashi, T., et al. 1999. Ischemia- and cytokineinduced mobilization of bone marrow-derived endothelial progenitor cells for neovascularization. Nat. Med. 5:434-438.

28. Rehman, J., Li, J., Orschell, C.M., and March, K.L. 2003. Peripheral blood "endothelial progenitor cells" are derived from monocyte/macrophages and secrete angiogenic growth factors. Circulation. 107:1164-1169.

29. Hong, Y.K., et al. 2002. Prox1 is a master control gene in the program specifying lymphatic endothelial cell fate. Dev. Dyn. 225:351-357.

30. van Rooijen, N., Bakker, J., and Sanders, A. 1997. Transient suppression of macrophage functions by liposome-encapsulated drugs. Trends Biotechnol. 15:178-185.

31. Biewenga, J., et al. 1995. Macrophage depletion in the rat after intraperitoneal administration of liposome-encapsulated clodronate: depletion kinetics and accelerated repopulation of peritoneal and omental macrophages by administration of Freund's adjuvant. Cell Tissue Res. 280:189-196.

32. van Rooijen, N., Kors, N., van der Ende, M., and Dijkstra, C.D. 1990. Depletion and repopulation of macrophages in spleen and liver of rat after intravenous treatment with liposome-encapsulated dichloromethylene diphosphonate. Cell Tissue Res. 260:215-222.

33. Oh, S.J., et al. 1997. VEGF and VEGF-C: specific induction of angiogenesis and lymphangiogenesis in the differentiated avian chorioallantoic membrane. Dev. Biol. 188:96-109.

34. Karkkainen, M.J., et al. 2003. Vascular endothelial growth factor $\mathrm{C}$ is required for sprouting of the first lymphatic vessels from embryonic veins. Nat. Immunol. 5:74-80.

35. Cao, R., et al. 2004. PDGF-BB induces intratumoral lymphangiogenesis and promotes lymphatic metastasis. Cancer Cell. 6:333-345.

36. Sonoda, Y., and Streilein, J.W. 1992. Orthotopic corneal transplantation in mice--evidence that the immunogenetic rules of rejection do not apply. Transplantation. 54:694-704.

37. Chang, L., Kaipainen, A., and Folkman, J. 2002. Lymphangiogenesis new mechanisms. Ann. N. Y. Acad. Sci. 979:111-119.

38. Schlaeger, T.M., Qin, Y., Fujiwara, Y., Magram, J., and Sato, T.N. 1995. Vascular endothelial cell lineage-specific promoter in transgenic mice. Development. 121:1089-1098.

39. Puri, M.C., Rossant, J., Alitalo, K., Bernstein, A., and Partanen, J. 1995. The receptor tyrosine kinase TIE is required for integrity and survival of vascular endothelial cells. EMBO J. 14:5884-5891.

40. Ambati, J., et al. 2003. An animal model of agerelated macular degeneration in senescent $\mathrm{Ccl}-2$ - or Ccr-2-deficient mice. Nat. Med. 9:1390-1397.

41. Takeuchi, M., Alard, P., and Streilein, J.W. 1998. TGF-beta promotes immune deviation by altering accessory signals of antigen-presenting cells. J. Immunol. 160:1589-1597. 